\title{
Front-End of Yb-based High-Energy Optical Waveform Synthesizer
}

\section{Hüseyin Çankaya, Anne-Laure Calendron, Giovanni Cirmi, and Franz X. Kärtner}

Center for Free-Electron Laser Science, Deutsches Elektronen Synchrotron (DESY), Notkestrasse 85, 22607 Hamburg, Germany

The Hamburg Centre for Ultrafast Imaging and Department of Physics, Universität Hamburg, Luruper Chaussee 149, 22761 Hamburg, Germany

Department of Electrical Engineering and Computer Science and Research Laboratory of Electronics, Massachusetts Institute of Technology, Cambridge, Massachusetts 02139, USA 


\section{Motivation}

- Challenge 1: Generation of Isolated Attosecond-pulse

- Solutions:

- Using gating techniques:

- Polarization gating

- Double-optical gating

- Ionization gating

- Two-color driving

- Using single or sub-cycle driver pulses

- Challenge 2: Improvement of efficiency

- Optical efficiency is on the order of 10-5-7

- Solution: Phase matching optimization and short-wavelength driver laser

- Challenge 3: Extend x-ray cut-off

- Generation of x-ray in water window.

- Solution: Long-wavelength driver laser

[1] G. Sansone, et al., Science 314(5798), 443-446 (2006).

[2] H. Mashiko, et al. Phys. Rev. Lett. 100(10), 103906 (2008)

[3] M. J. Abel, et al. Chem. Phys. 366(1-3), 9-14 (2009).

[4] B. Kim, et al. Opt. Express 16(14), 10331-10340 (2008).

[5] Chipperfield et al., PRL 102, 063003 (2009) 


\section{Driver Laser Requirements}

- Broadband laser pulses supporting single or sub-cycle pulses
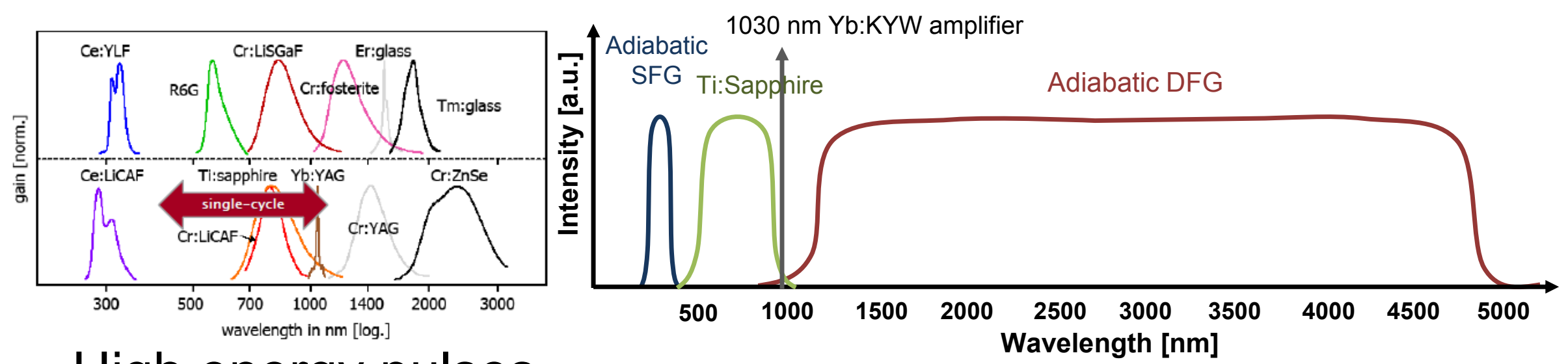

- High energy pulses

- Carrier-envelope phase stability
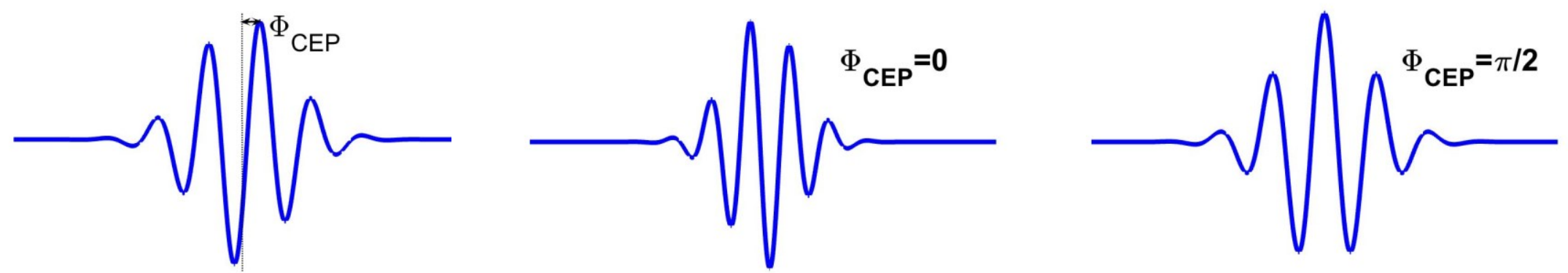


\section{Suggested Laser Source}

\section{- Optical Wave Parametric Synthesizer}

- Generation of broadband seed source

- Amplify different spectral regions separately in a optical parametric amplifier

- Combine and compress

Adjustment of timing in sub-cycle time duration Control of relative phase

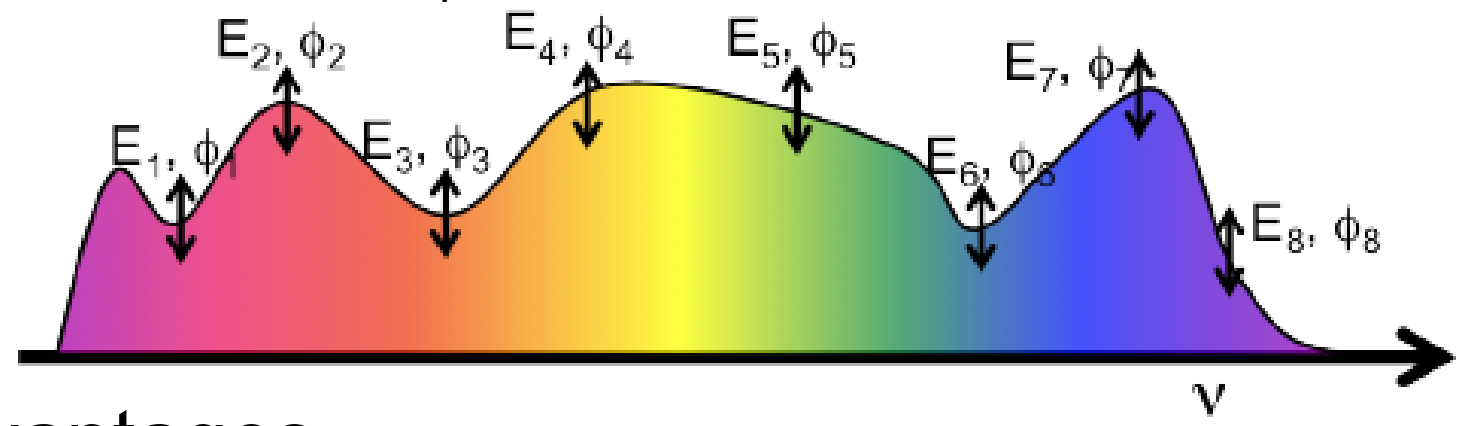

- Advantages

- Energy scalability

- Spectral scalability

- Control in electric field of the driver laser 


\section{Previous works}

- $\mathrm{mJ}$ pulses compressed in hollow core fibers from sub-50 fs to few-cycle duration [1]

A

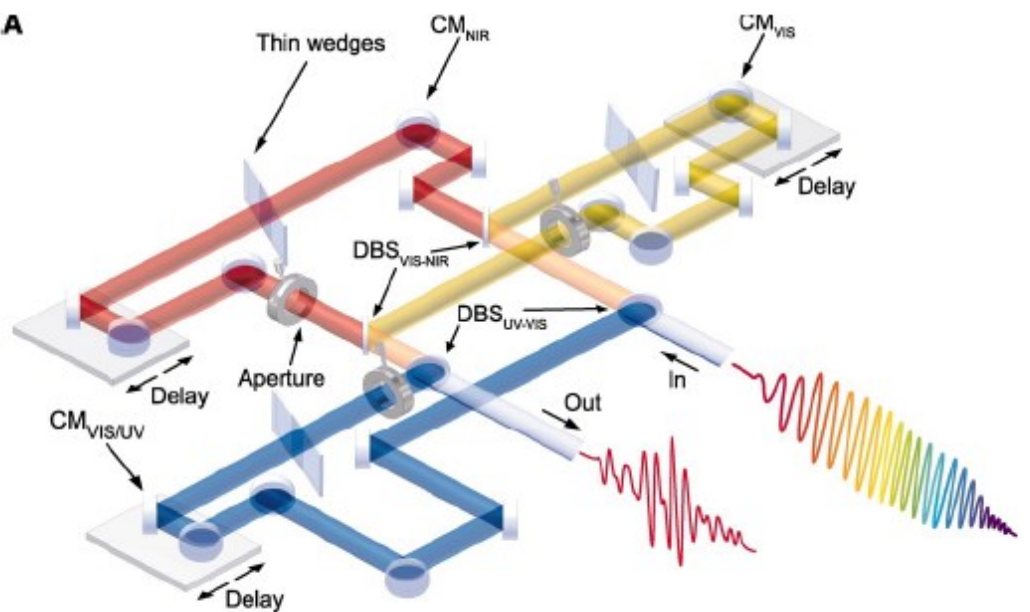

- Serial approach: in one arm, amplification of the spectral regions one after each other [2]

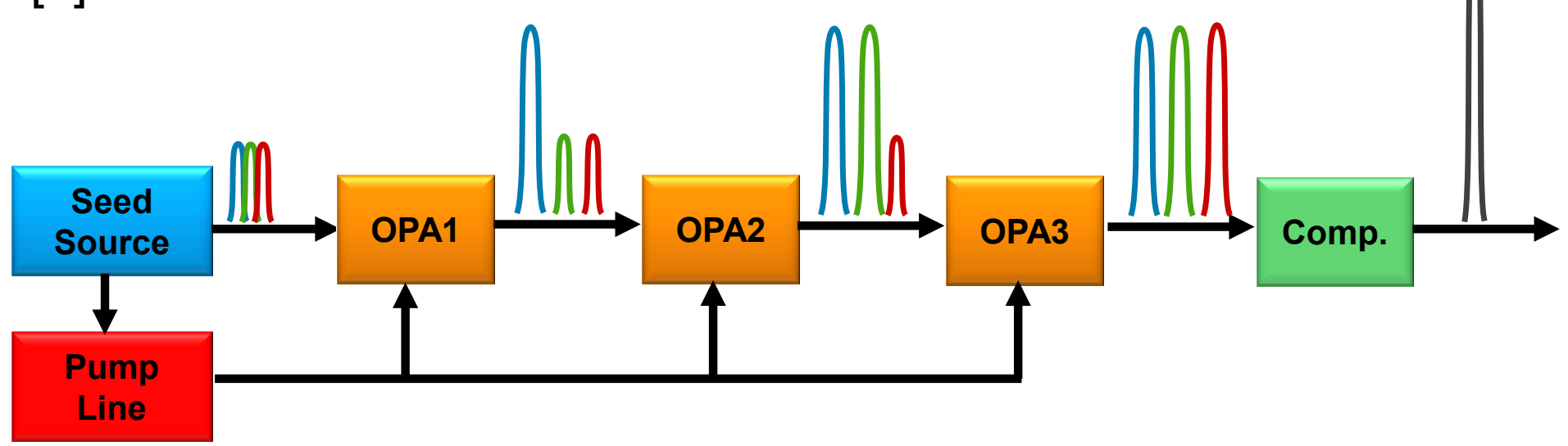

[1] Wirth et al., Science 34, 195, (2011)

[2] Harth et al., OE 20, 3076, (2012) 


\section{Previous works}

Parallel approach: each amplification arm amplifies only one spectral region

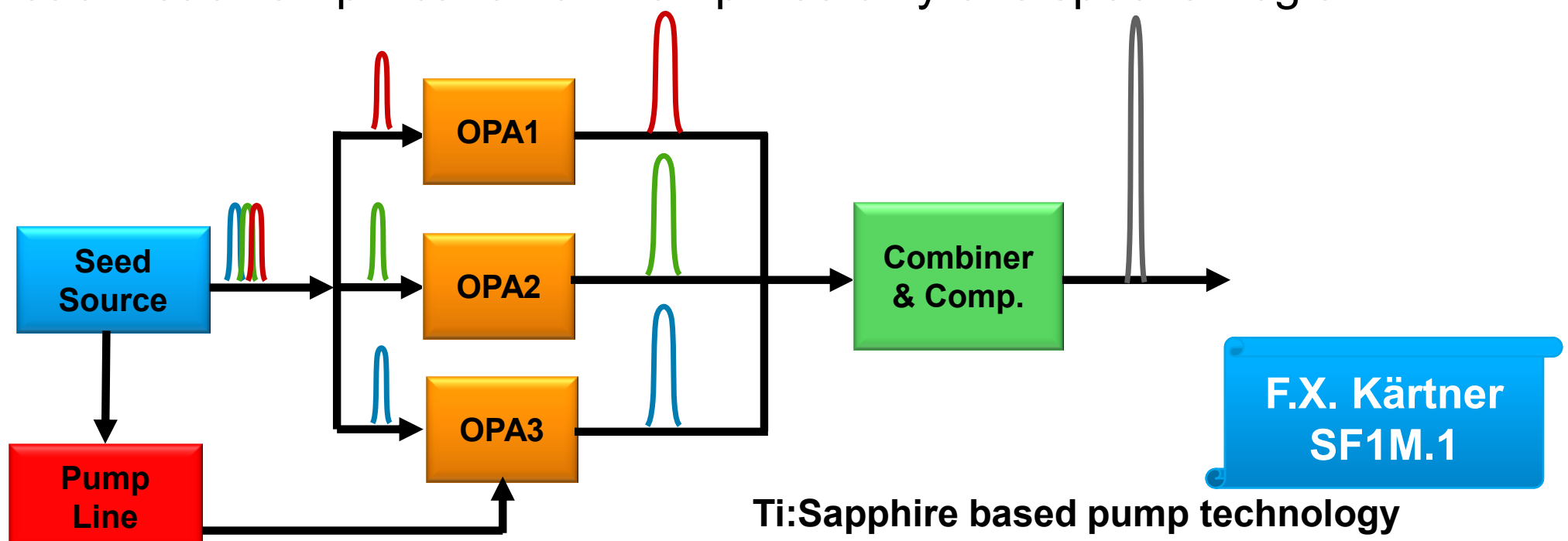

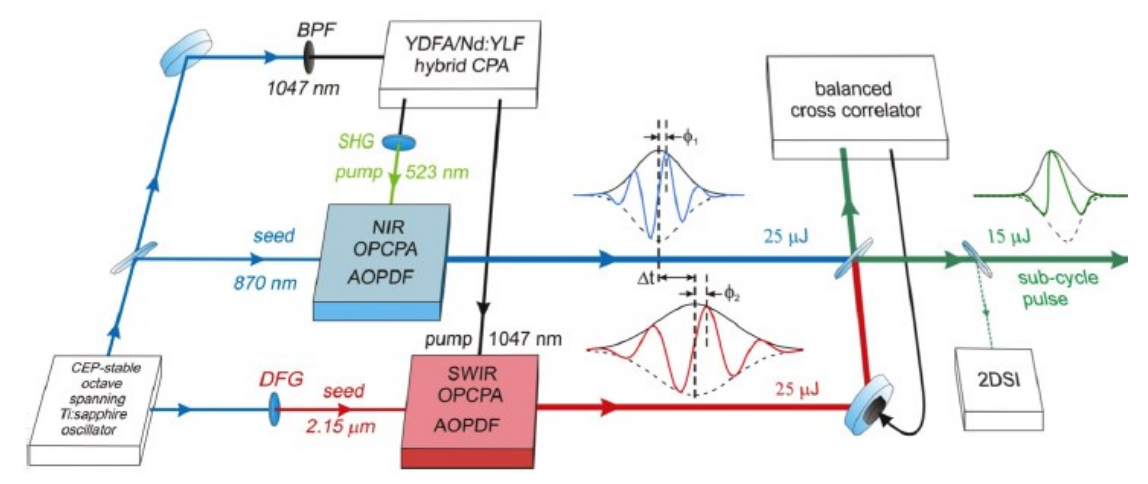

Huang et al., Nature photonics, 5, 475 (2011)

Hong et al. Optics Letters, 39, 3145 (2014)

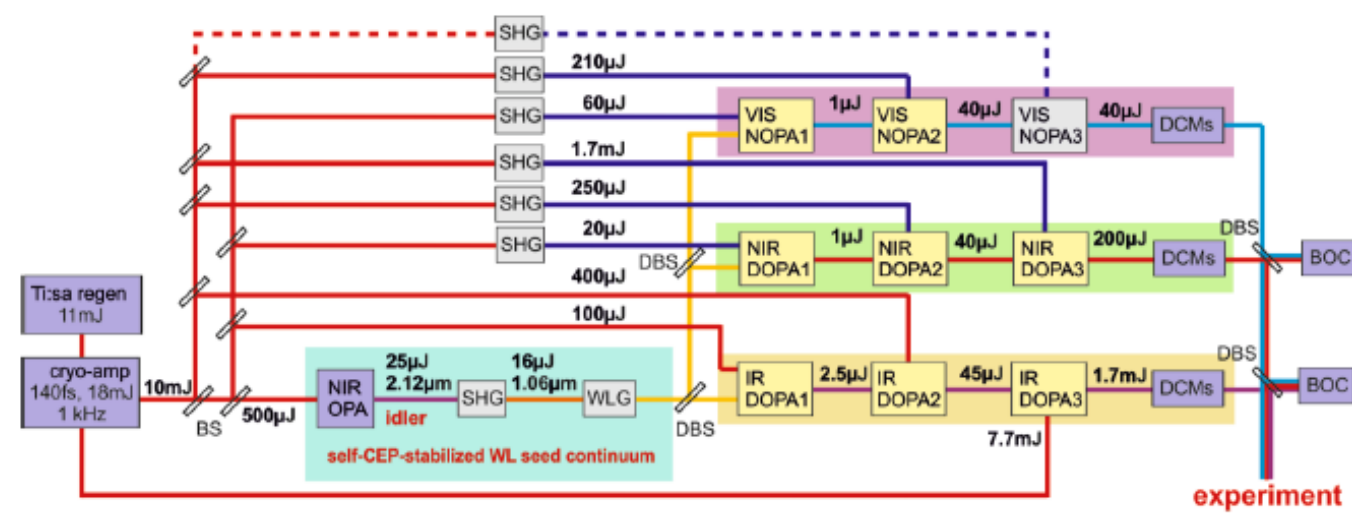

Cirmi et al., UFO IX, We3.3, (2013)

Manzoni et al, Laser\&Photonics Review (2015) 


\section{Our Planned System}

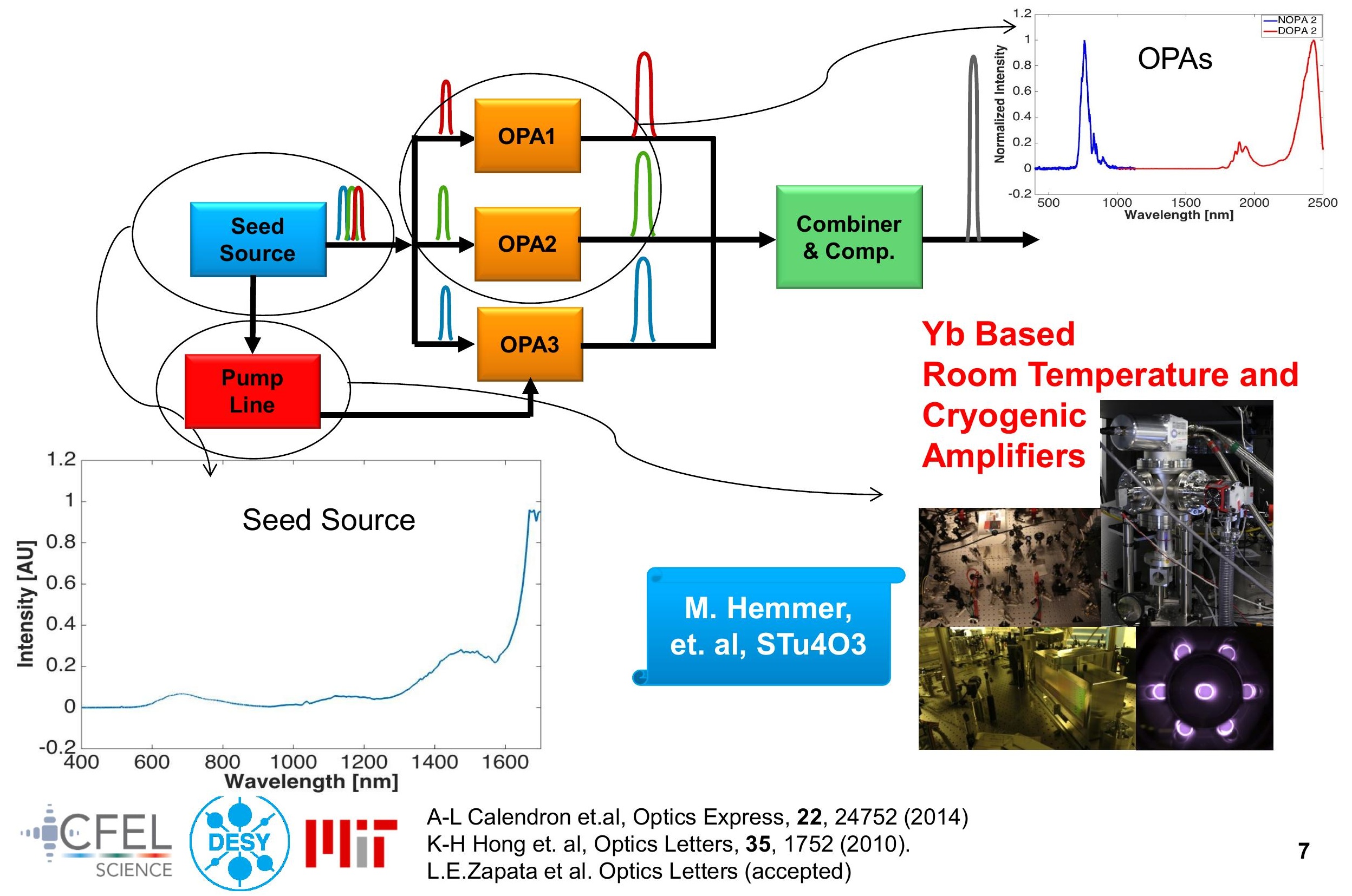




\section{Passively CEP Stable Seed Source}

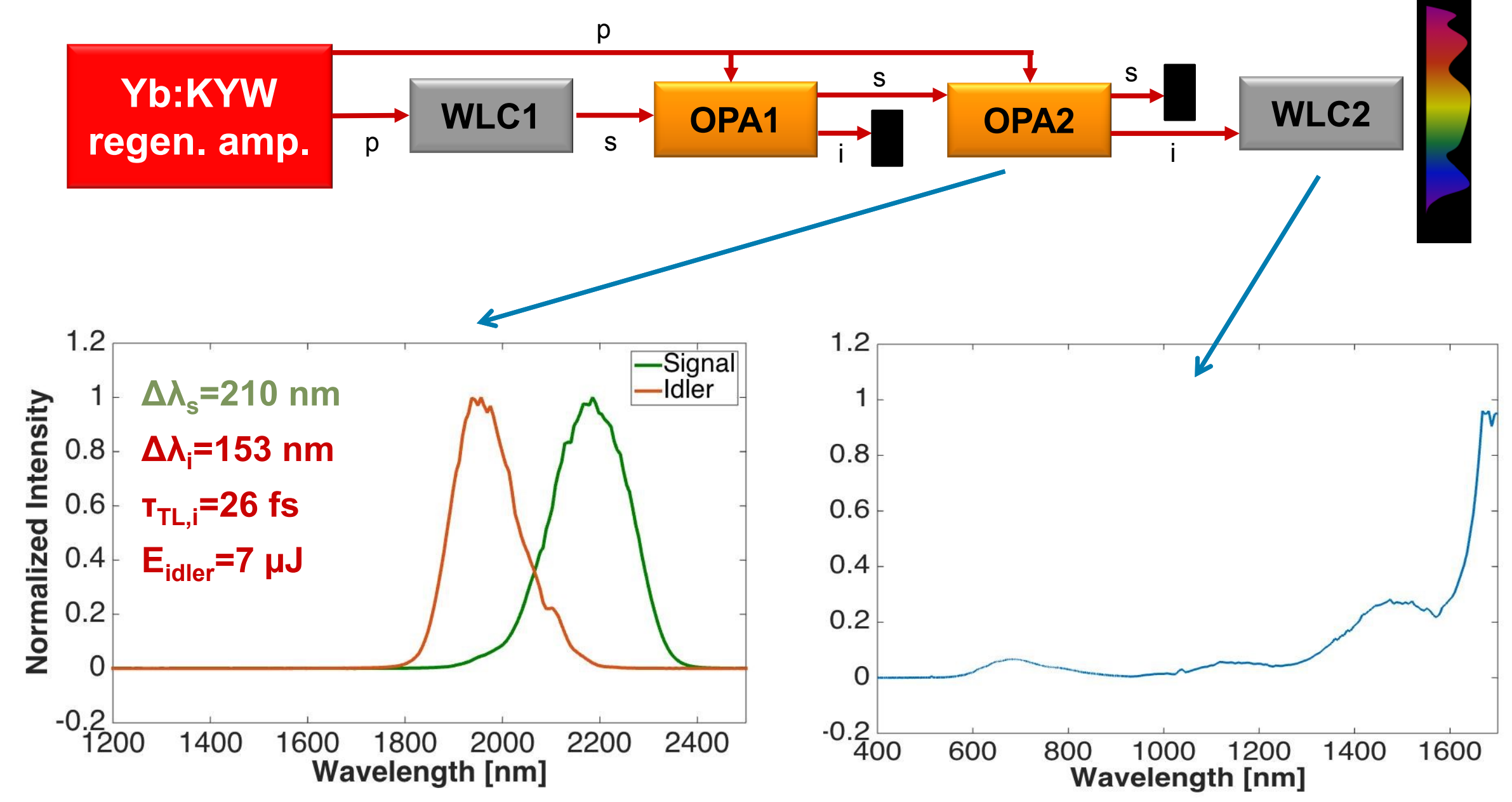




\section{CEP Characterization}
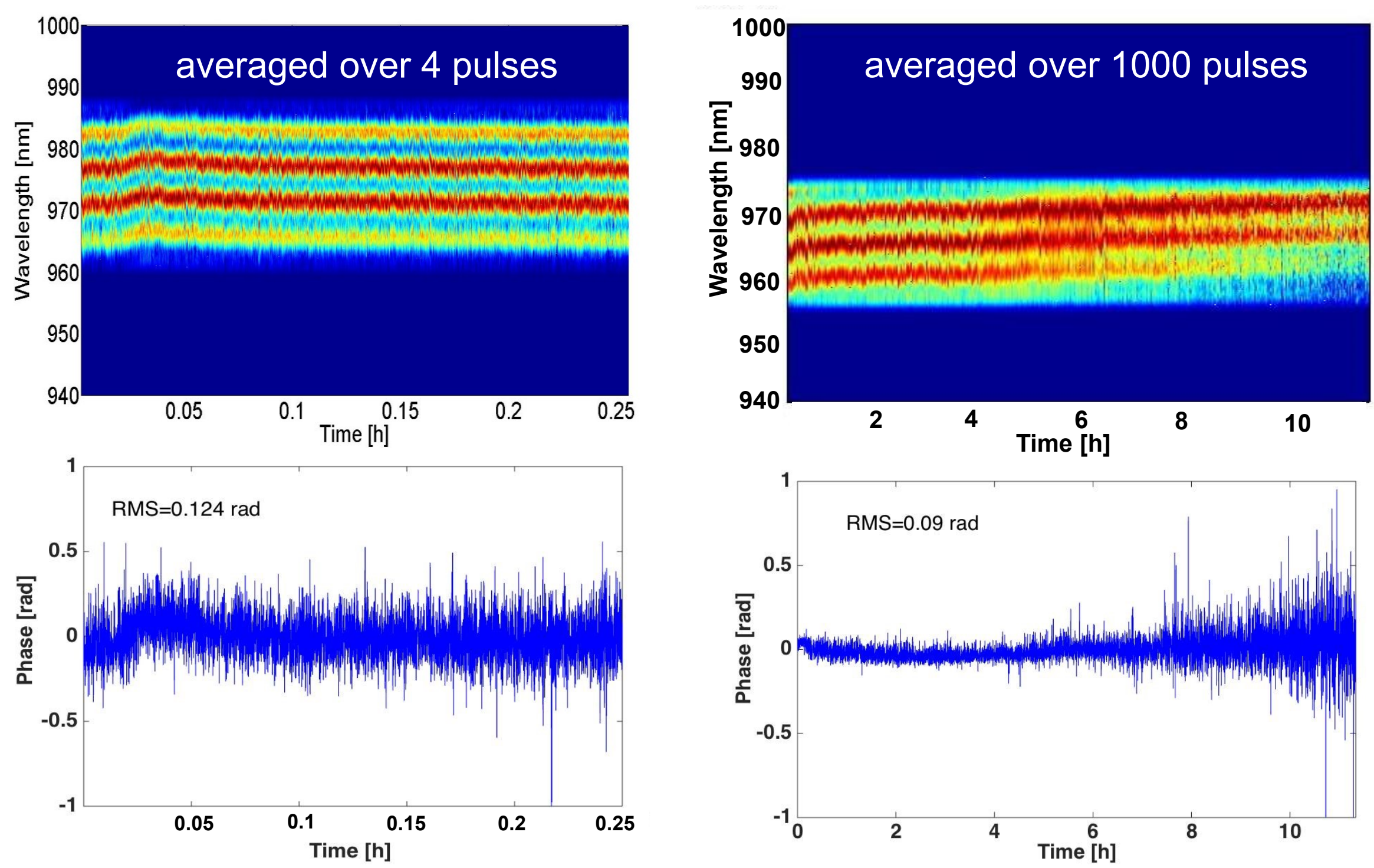

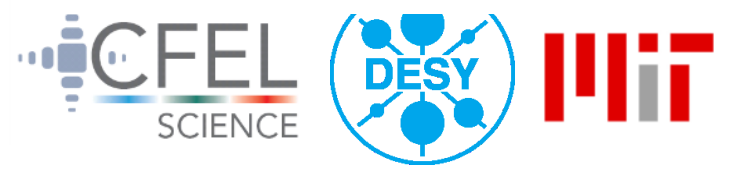

H. Cankaya, A-L Calendron, F.X. Kärtner., Ultrafast Phenomena, Okinawa, (2014) 


\section{Modification in CEP Stable Seed Source}

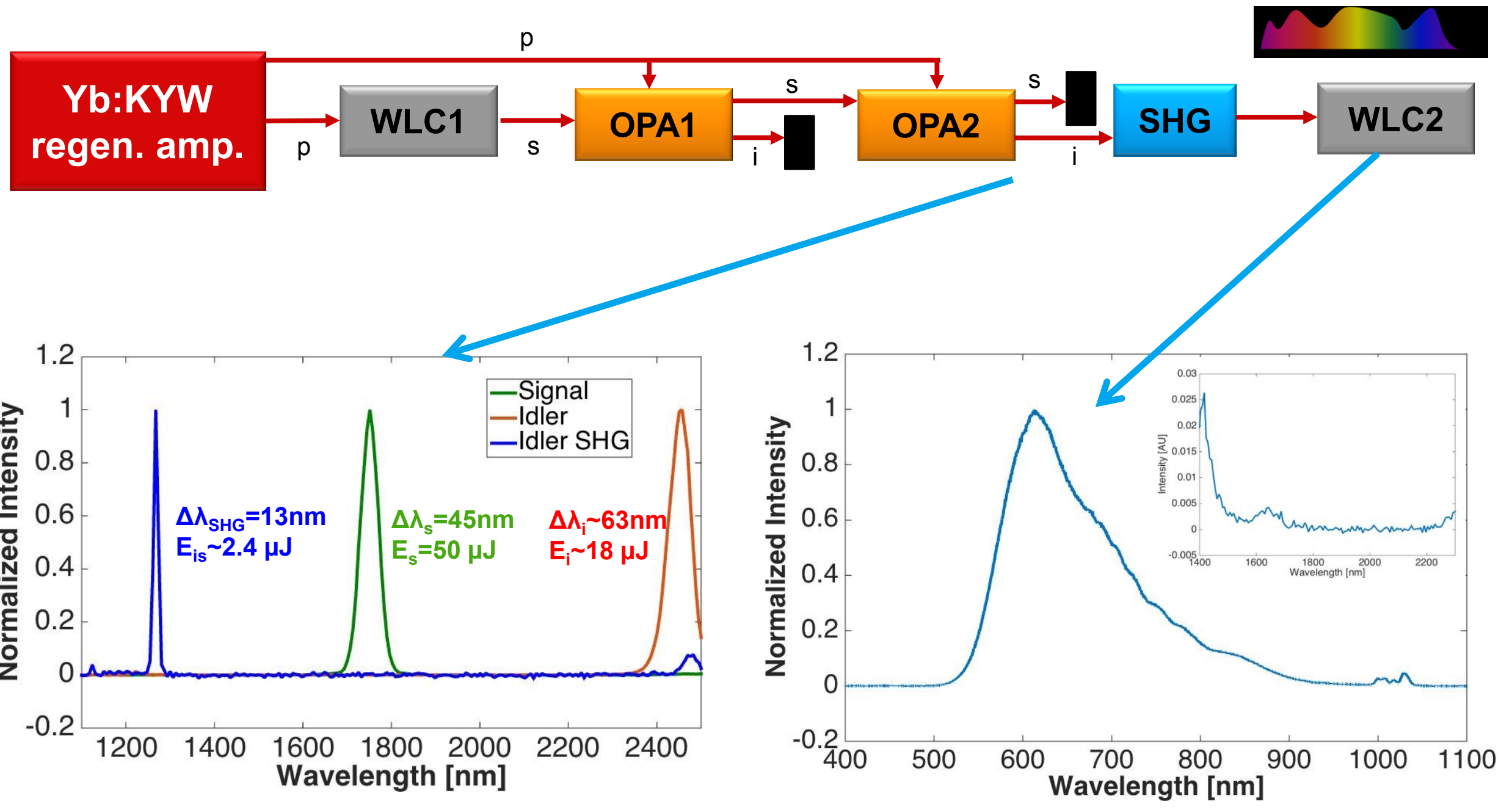




\section{4-Hour Operation of Seed Source}

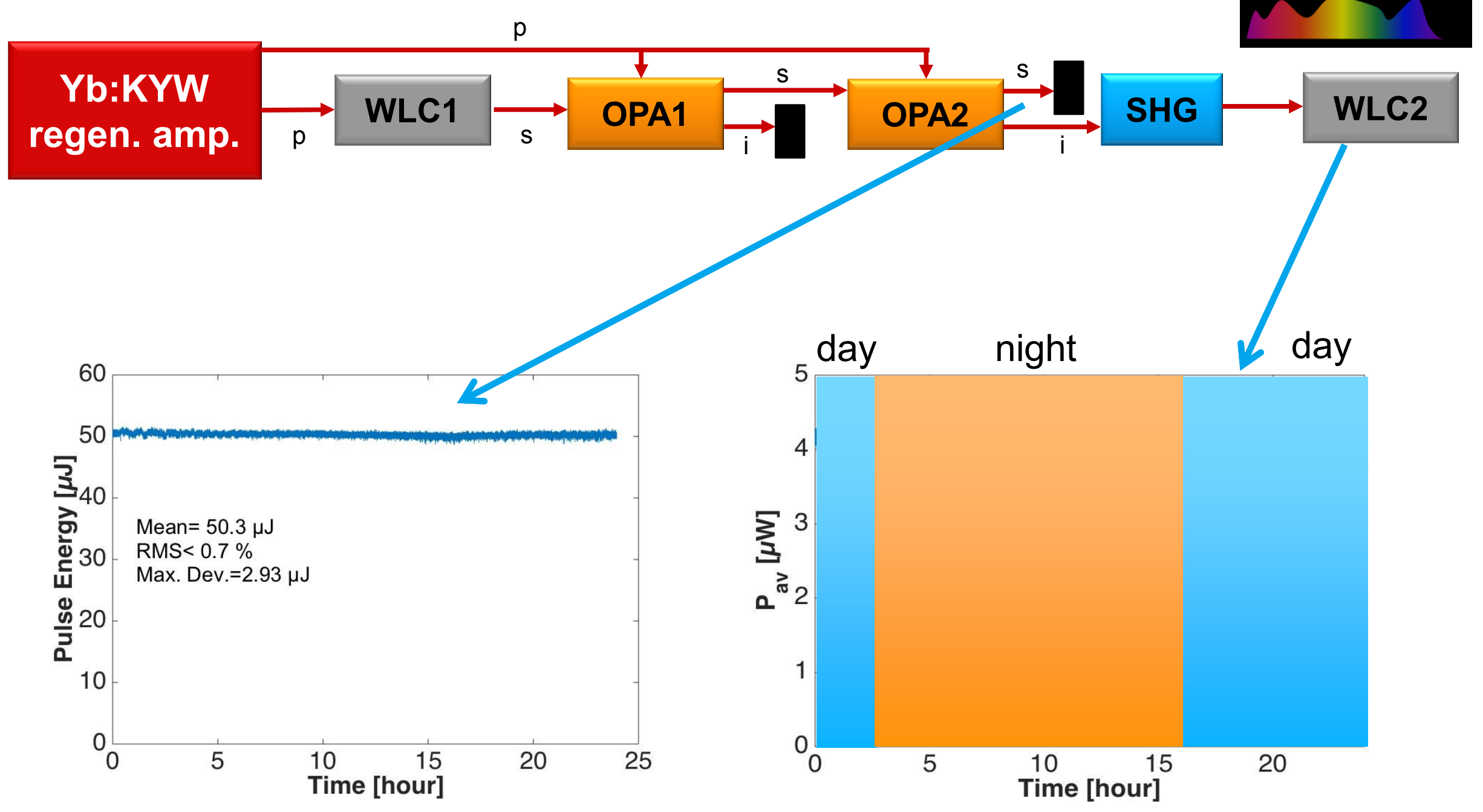




\section{Two Channel OPAs}
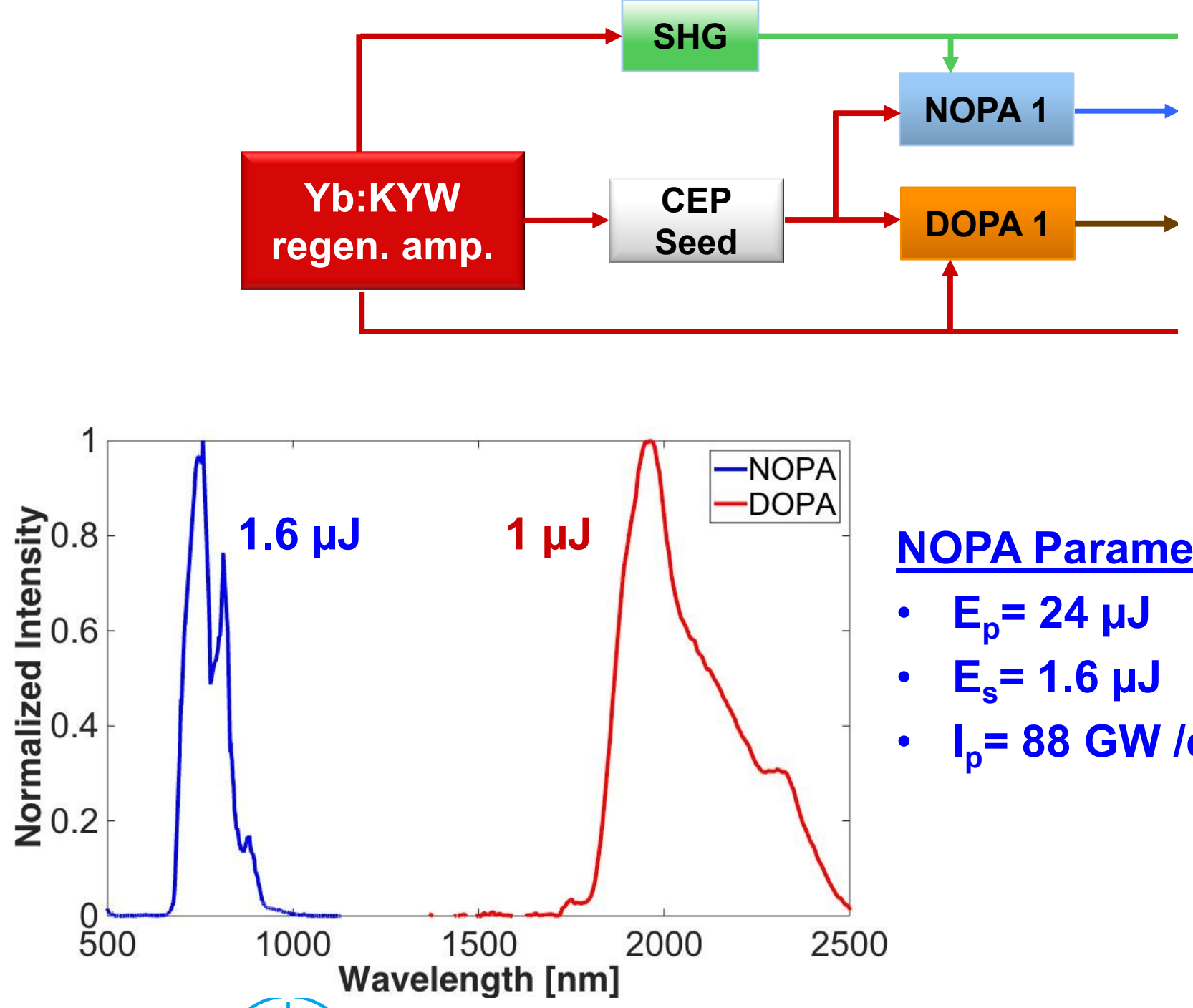

NOPA Parameters: DOPA Parameters:

- $\mathrm{E}_{\mathrm{p}}=24 \mu \mathrm{J}$

- $E_{\mathrm{s}}=1.6 \mu \mathrm{J}$

- $I_{p}=88 \mathrm{GW} / \mathrm{cm}^{2}$
- $E_{p}=105 \mu \mathrm{J}$

- $E_{\mathrm{s}}=1 \mu \mathrm{J}$

- $I_{p}=130 \mathrm{GW} / \mathrm{cm}^{2}$ 


\section{Two Channel OPAs}
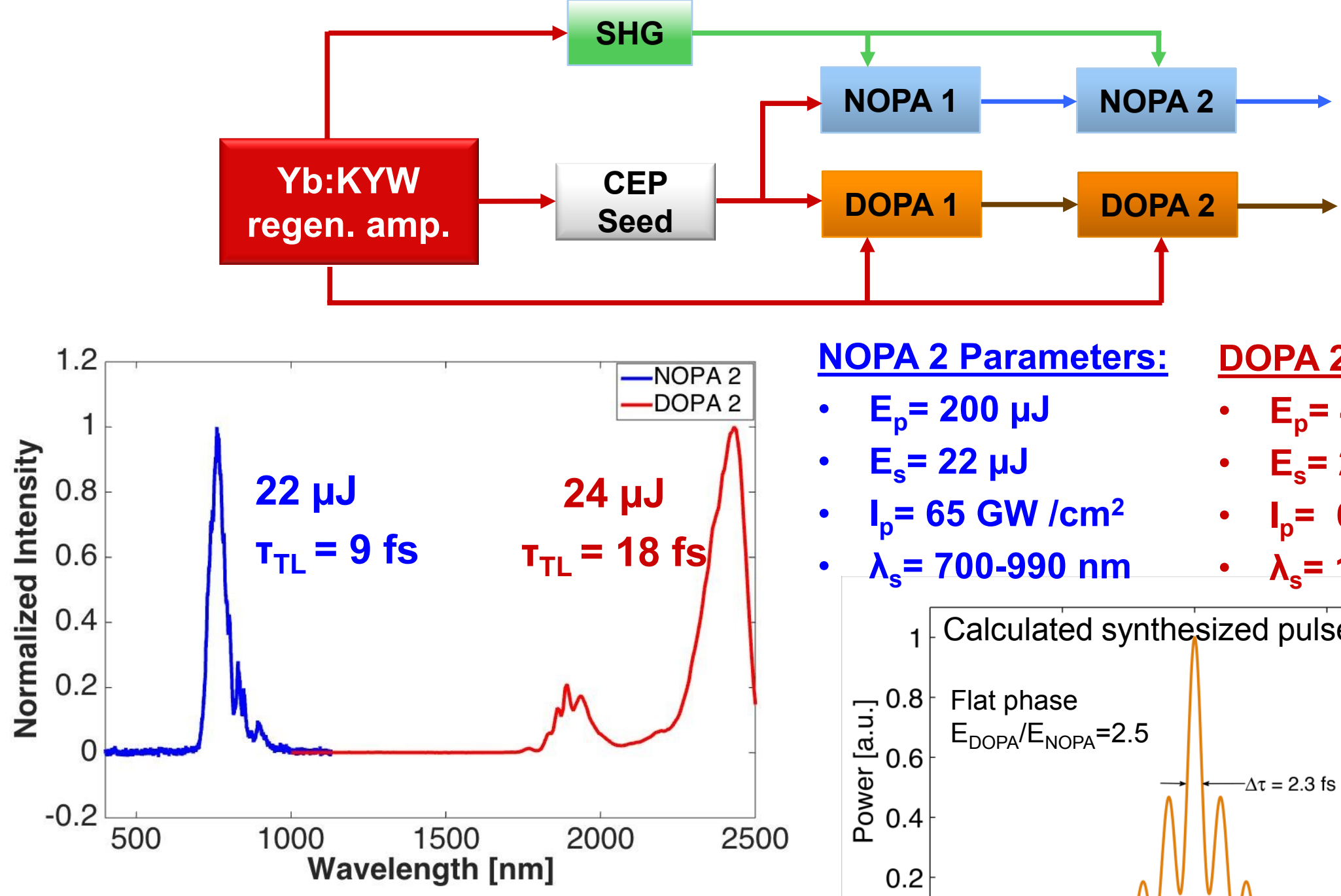

NOPA 2 Parameters: DOPA 2 Parameters:

- $E_{p}=200 \mu J$

- $E_{s}=22 \mu \mathrm{J}$

- $\mathrm{I}_{\mathrm{p}}=65 \mathrm{GW} / \mathrm{cm}^{2}$

- $\lambda_{\mathrm{s}}=700-990 \mathrm{~nm}$
- $E_{p}=470 \mu \mathrm{J}$

- $E_{s}=24 \mu \mathrm{J}$

- $I_{p}=69 \mathrm{GW} / \mathrm{cm}^{2}$

- $\lambda_{\mathrm{s}}=1800-2500 \mathrm{~nm}$

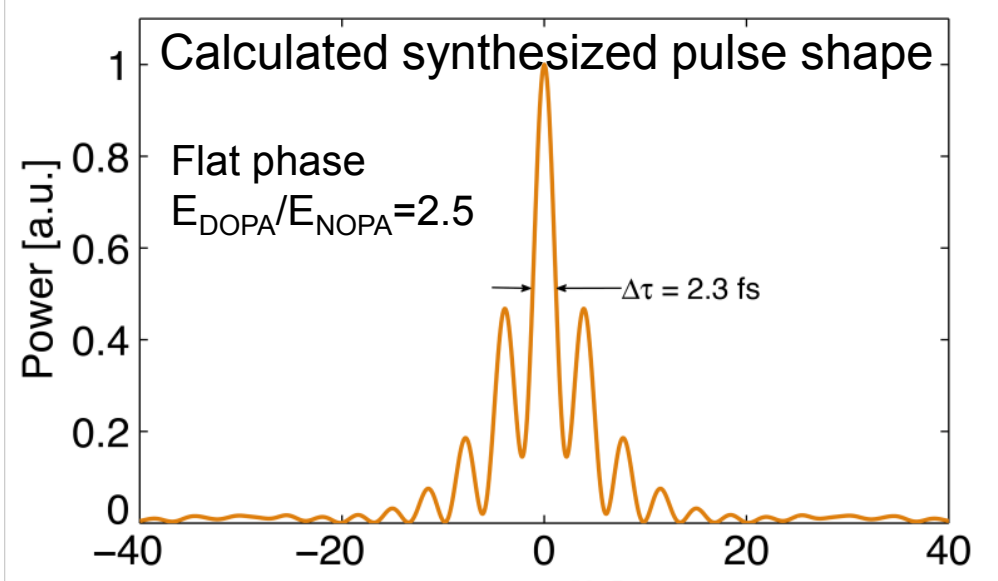

A-L Calendron, H. Cankaya,

Time [fs] 


\section{Summary and Outlook}

\section{Summary:}

- CEP stable seed source based on Yb pump laser

- CEP stability less than 100 mrad for 11 hour operation

- Two-channel OPA stages 20- $\mu \mathrm{J}$ pulses per channel

\section{Outlook:}

- Amplification to mJ level

- Characterization of spectral-phase and compression

- Pulse synthesis
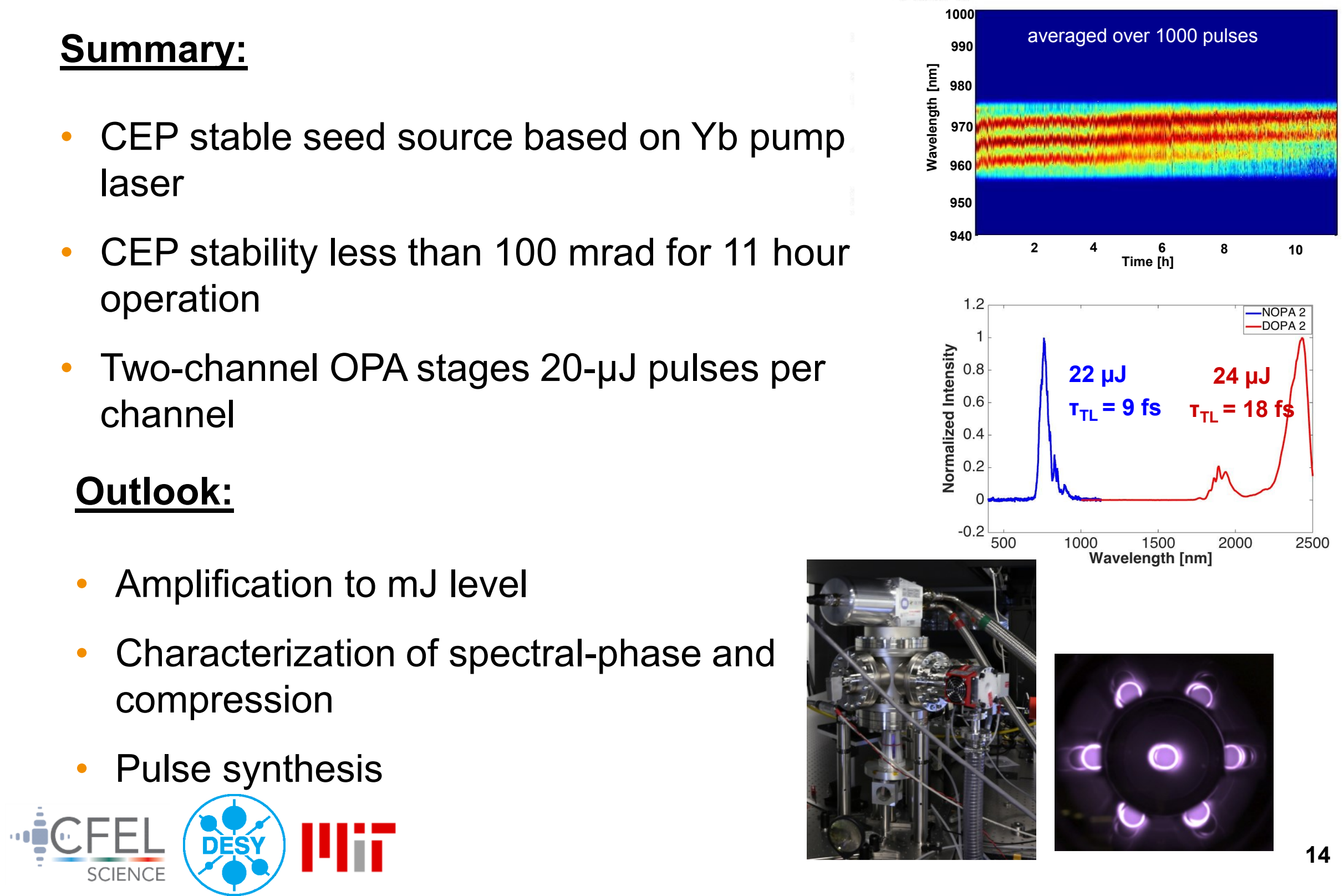


\section{Acknowledgements}
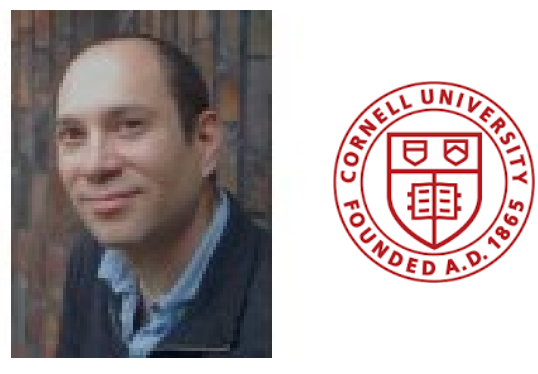

Jeff Moses

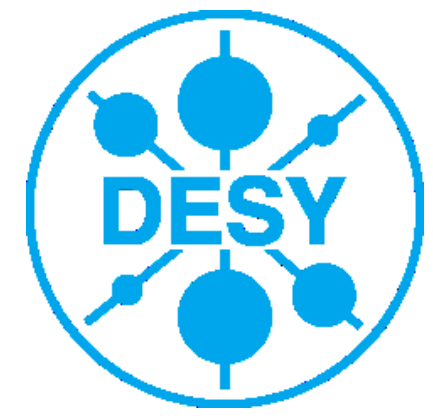

(IIIFEL
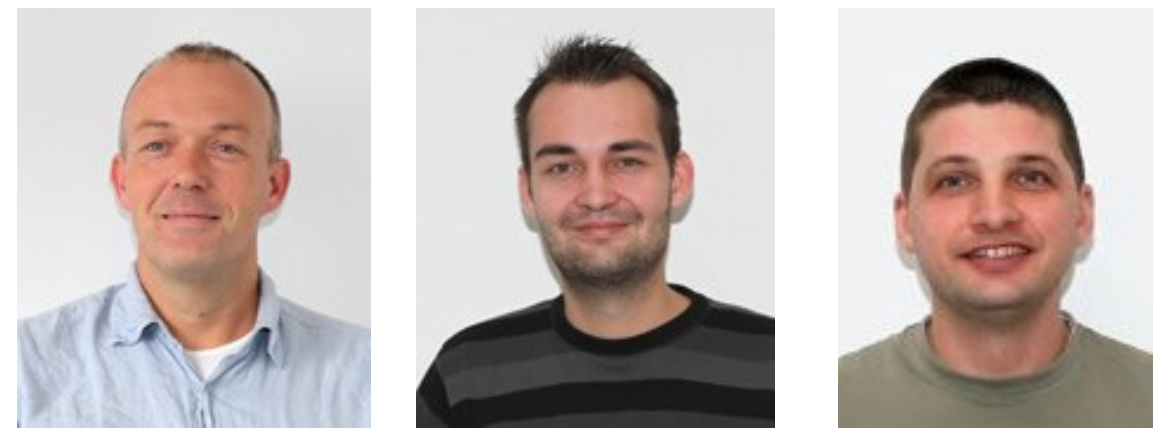

CFEL Engineering Team

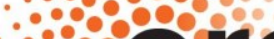

$\therefore \because \because 08 \cap$

$\therefore \because 010$

$\because 0 \%$

$\because \because \because \because \bullet$

European Research Council

Established by the European Commission

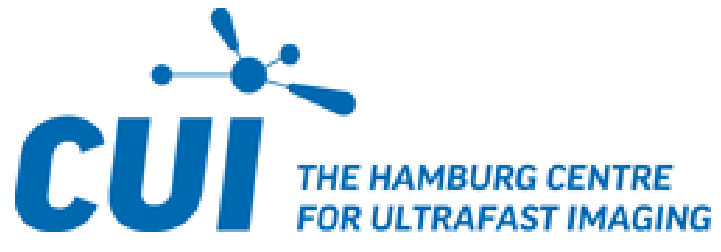




\section{Thank you \\ for your attention}

